\title{
Will the real angiotensin converting enzyme please stand up?
}

\author{
Friedrich C. Luft
}

Published online: 10 March 2012

(C) Springer-Verlag 2012

The angiotensin converting enzyme (ACE) is a $180-\mathrm{kD}$ expopeptidase with two homologous domains that catalyzes the conversion of angiotensin I (Ang I) to angiotensin II (Ang II), a potent vasoconstrictor, in a substrate concentrationdependent manner. ACE also degrades bradykinin, an important vasodilator, as well as other vasoactive peptides. Angiotensin I converting enzyme 2 (ACE-2) is an exopeptidase that catalyzes the conversion of angiotensin I to the nonapeptide angiotensin (1-9) or the conversion of angiotensin II to angiotensin (1-7). Ang II interacts with two receptors; the AT1 receptor performs the well-appreciated vasoconstrictor, aldosterone-releasing, and sodium-reabsorptive activities. The AT2 receptor seems to perform ameliorative or other unknown functions. Ang (1-7) has also been implicated in cardioprotection and interacts with the Mas receptor, encoded by a proto-oncogene of the same name (Fig. 1). Subsequent signaling is also imperfectly defined.

We know quite a bit about ACE. The Bernstein laboratory performed a series of novel strategic promoter-swapping studies indicating that ACE plays a role in many other physiologic processes beyond simple blood pressure control [1]. ACE-2, an ACE homolog with about a $40 \%$ homology and only one active catalytic domain, was cloned in 2000. ACE-2 has direct effects on cardiac function and is expressed predominantly in vascular endothelial cells, the heart, the kidneys, and testis [2]. Then, there is the more ethereal ACE-3 that is an interacting protein in sperm with the IZUMO1 protein. The interrelationship apparently facilitates sperm-egg interaction. In man, ACE3 apparently only exists as a pseudo-gene with no functional

F. C. Luft $(\bowtie)$

Experimental and Clinical Research Center, Charité Medical Faculty and Max-Delbrück Center for Molecular Medicine, Lindenbergerweg 80,

13125 Berlin, Germany

e-mail: luft@charite.de relevance [3, 4]. ACE and ACE-2 are absent in the Drosophila. However, this invertebrate has homologous sequences probably belonging to a different family, an angiotensin-related converting enzyme (ACER). The ACER proteins are active endopeptidases important during fly development but seem to lack a clear function in adulthood [5]. Bacteria also contain an ACE-like protein that cleaves Ang I to Ang II, as shown for Xanthomonas axonopodi [6]. The findings suggest that the ACEs have been around far longer than the angiotensins. Furthermore, ACE-2 seems to be senior to ACE.

ACE-2 won our hearts over with the finding that in hypertensive rat strains, ACE-2 messenger RNA and protein expression were markedly reduced. Targeted disruption of ACE-2 in mice resulted in a severe cardiac contractility defect, increased Ang II levels, and upregulation of hypoxia-induced genes in the heart. Genetic ablation of ACE on an ACE-2-mutant background rescued the cardiac phenotype. Interestingly, disruption of ACER, the Drosophila ACE-2 homologue, resulted in fruit fly heartbreak [7]. These genetic data for ACE-2 showed that it is an essential regulator of cardiac function in vivo. A second independent ACE-2 gene deletion story gave substantially less dramatic results, but nevertheless "hope springs eternal" [8].

Then, there is Hartnup disease. When I was a medical student (50 years ago), I encountered a family with this "pellagra-like" dermatosis. We knew at the time that the problem lay in the failed absorption of tryptophan and other non-polar amino acids. Tryptophan is necessary to make serotonin, melatonin, and niacin. The Hartnup amino acid transporter (SLC6A19) is the major luminal sodiumdependent neutral amino acid transporter of small intestine and renal proximal tubule. SLC6A19 renal expression depends on its association with collectrin (Tmem27), a protein homologous to the membrane-anchoring domain of ACE-2. And so, Camargo et al. [9] showed that ACE-2 is 


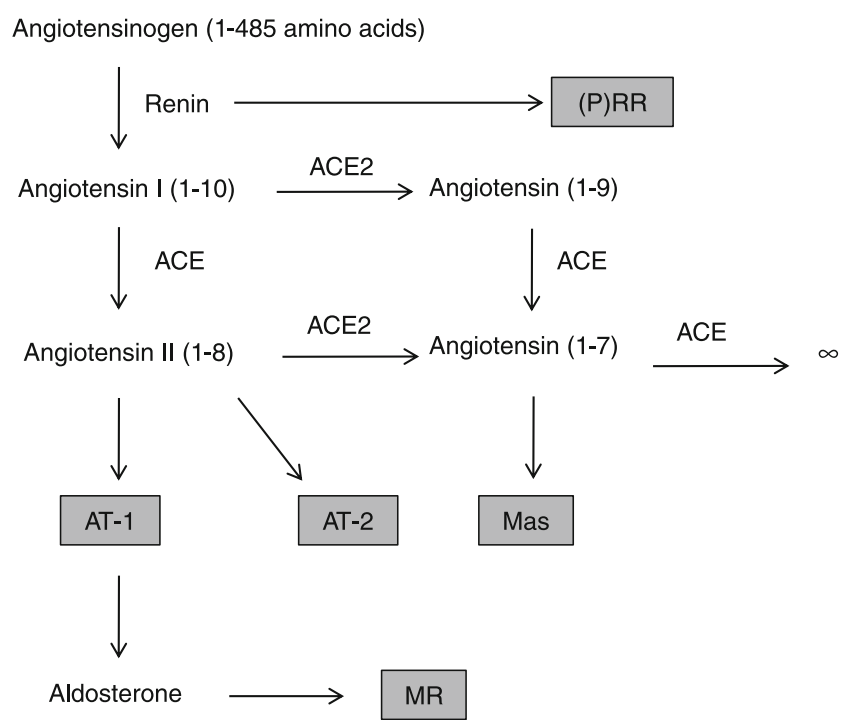

Fig. 1 The aspartyl protease renin cleaves angiotensinogen to the ten amino acid peptide, Ang I. The angiotensin converting enzyme ( $A C E)$ in turn cleaves Ang I to the eight amino acid peptide, Ang II. Ang II can interact with two receptors (AT1 and AT2) that appear to have divergent functions. Ang I and Ang II can be cleaved to smaller products by a second angiotensin converting enzyme $(A C E-2)$. One of these small products (Ang 1-7) can interact with its own receptor termed Mas

necessary for the expression of the Hartnup transporter in intestine and suggested that the differential functional association of mutant SLC6A19 transporters with ACE-2 and collectrin in intestine and kidney participate in the phenotypic heterogeneity of Hartnup disease.

Several years ago, a coronavirus $(\mathrm{CoV})$ that can unleash a lethal severe adult respiratory distress syndrome (SARS) caused worldwide panic. Subsequently, Kuba et al. [10] provided genetic evidence that ACE-2 serves as a SARS receptor. ACE-2 gene-deleted mice were resistant to virus infection. SARS-CoV infections and the Spike protein of the
SARS-CoV also reduced the ACE-2 expression. Notably, injection of SARS-CoV Spike into mice worsened acute lung injury (ALI) in vivo, which was attenuated by blocking the renin-angiotensin system pathway. These results provided a molecular explanation why SARS-CoV infections caused severe and oftentimes lethal ALI. Downregulation of ACE-2 by SARS-CoV could accelerate the SARS pathogenesis. Furthermore, the results suggested a possible therapy for SARS and other respiratory disease viruses that elicit ALI. All these findings are fascinating but confusing. First, we are dealing with a matrix metalloproteinase that has been around a very long time from bacteria, to invertebrates, and to vertebrates. The findings imply a role in cardiovascular regulation from invertebrates to vertebrates. Furthermore, a simple conversion of Ang I to Ang II or degradation of bradykinin cannot be the end of the story. We are left with "rather messy" renin-angiotensin-aldosterone system remains that include Ang (1-9), Ang (1-7), the Mas proto-oncogene, and other unfathomable variables. Then, we skirt by Hartnup disease and collectrin to learn that ACE-2 embraces fundamental processes in gastrointestinal and renal tubular reabsorption. Are these functions of ACE2 in any way related to Ang (1-7)? Could there be other substrates, and if so, what are the substrates and what are the end products? Or perhaps the molecule has still another function? All that information should be reason for a major migraine, but there is more to come.

In this issue, Rey-Parra et al. hypothesized that ACE-2 prevents bleomycin-induced lung injury. Bleomycin belongs to a family of chemotherapeutic agents that induce DNA strand breaks. When I was a junior physician, we included bleomycin in our regimen for patients with Hodgkin's lymphoma. Pulmonary toxicity was common - a model of drug-induced pulmonary fibrosis in man. Rey-Parra et al. [11] studied mice and found that ACE-2 gene deletion worsens bleomycin-induced lung injury and more so in males than females (so much for the
Fig. 2 ACE-2 has protective functions in lung that are complex. Some protection may be afforded by generating Ang (1-7) that signals via the Mas receptor. ACE-2 is also a receptor for the $\mathrm{CoV}$ responsible for SARS (ARDS) an ALI. CoV after cell entry downregulates ACE- 2 by unclear mechanisms. The Absence of ACE-2 increases susceptibility to ALI as induced by bleomycin in the work by Rey-Parra et al. [11]. Finally, ACE-2 is a homolog of an amino acid transporter.

Much needs to be learned about this complex signaling process (adapted from [13])

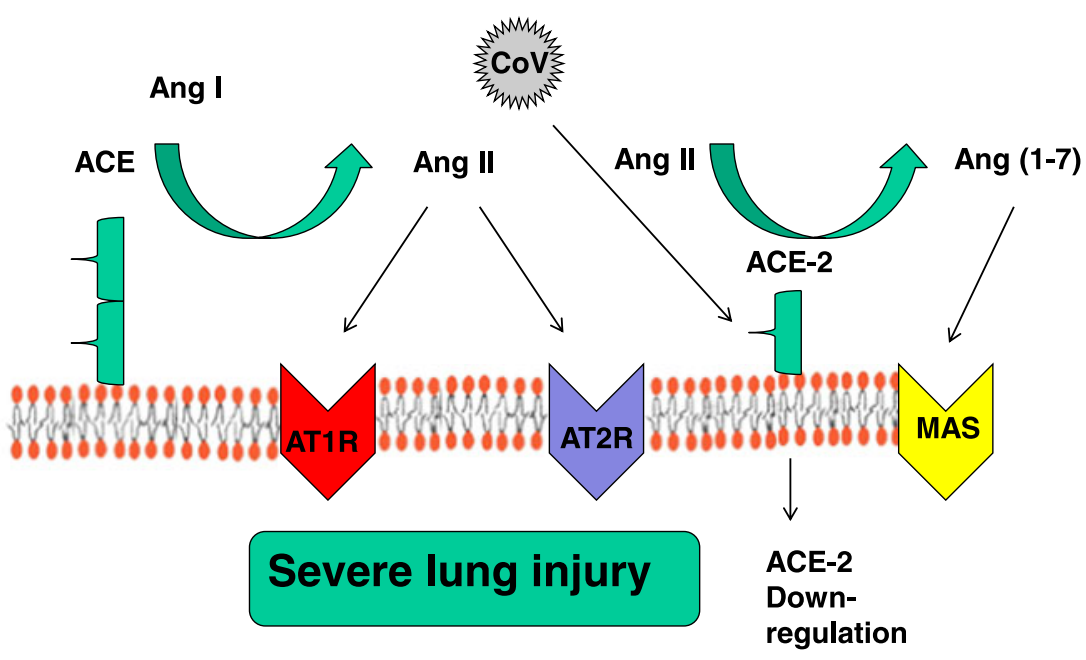

Bleomycin and other toxins 
myth of male privilege). Pulmonary fibrosis, TGF- $\beta 1$, collagen, and hydroxyproline expression were all more severe in ACE-2 gene-deleted mice, compared to controls. Furthermore, exercise tolerance and dynamic compliance were markedly reduced in male ACE-2 gene-deleted mice given bleomycin. In female ACE-2 gene-deleted mice, these effects were less pronounced. To apply ACE-2 therapeutically, the authors expressed the amino acid residues 1-740 (rhACE-2) in Chinese hamster ovary cells. The rhACE-2 was administered intraperitoneally for 221 days at $2 \mathrm{mg} / \mathrm{kg}$. The ACE- 2 treatment protected against bleomycin-induced fibrosis; in female mice, this treatment caused a complete reduction in fibrosis. However, these experiments were performed in wild-type mice so that a rescue experiment in ACE-2 gene-deleted mice was evidently not performed. The authors next included some data on AT1 and AT2 receptor expression. These experiments were done in the female mice without gene deletion. The AT2 receptor expression appears to have been enhanced, particularly in female mice given bleomycin.

The pathogenesis of bleomycin-induced lung fibrosis is not well understood. Histological studies show that bleomycin treatment results in damage to endothelial cells in lung vasculature, followed by the accumulation of inflammatory cells, including macrophages and lymphocytes, in the interstitium. However, the mechanisms are still unclear. After bleomycin, lung fibroblast cells are activated by cytokines such as tumor necrosis factor and interleukins, and collagen is deposited in the alveolar space and capillaries. This chain of events results in reduced oxygen exchange and impaired lung function. Corticosteroids are sometimes prescribed to suppress the lung toxicity. The authors imply that the reninangiotensin system could participate in the bleomycin toxicity process and that ACE- 2 mediates protection. The Penninger laboratory reported earlier that ACE-2 and the AT2 receptor protected mice from severe ALI induced by acid aspiration or sepsis $[12,13]$. They showed that mice deficient for ACE show markedly improved disease, and also that recombinant ACE-2 (as demonstrated in the current report) protected mice from severe ALI (Fig. 2).

Rey-Parra et al. [11] suggest that recombinant ACE-2 could have therapeutic potential to attenuate respiratory morbidity in ALI and resultant acute respiratory distress syndrome (ARDS) from various causes. Can one matrix metalloproteinase with only one domain (as opposed to the two domains in ACE) do so many different things? First, what is the most important function of ACE-2 when ACE came later and ACE-3 exists in humans solely as a pseudogene? ACE-2 could counterbalance the effects of ACE (party-line thinking here) and possibly that state-of-affairs could justify why ACE-2 is around today. Second, it appears that ACE-2 plays a protective role in the risk of SARS-CoV, although ACE-2 is apparently necessary for CoV infection in the first place. The appearance of ACE-2 as a partner in transport systems is most fascinating. This finding is of major clinical relevance because aside from dramatic genetic diseases (Hartnup disease), these transporters have accrued relatively little attention. ALI and ARDS are exceptionally important. The idea of a recombinant ACE-2 therapy has appeal, although I could not quite follow how rhACE-2 moved so readily from the peritoneum into the lung. There is much work to be done in this field.

Respectfully,

Friedrich C. Luft

\section{References}

1. Shen XZ, Xiao HD, Li P, Lin CX, Billet S, Okwan-Duodu D, Adams JW, Bernstein EA, Xu Y, Fuchs S et al (2008) New insights into the role of angiotensin-converting enzyme obtained from the analysis of genetically modified mice. J Mol Med (Berl) 86 (6):679-684, Epub 2008 Apr 29

2. Imai Y, Kuba K, Ohto-Nakanishi T, Penninger JM (2010) Angiotensin-converting enzyme 2 (ACE2) in disease pathogenesis. Circ J 74:405-410

3. Inoue N, Kasahara T, Ikawa M, Okabe M (2010) Identification and disruption of sperm-specific angiotensin converting enzyme-3 (ACE3) in mouse. PLoS One 5:e10301

4. Evans JP (2012) Sperm-egg interaction. Annu Rev Physiol 74:477-502

5. Houard X, Williams TA, Michaud A, Dani P, Isaac RE, Shirras AD, Coates D, Corvol P (1998) The Drosophila melanogasterrelated angiotensin-I-converting enzymes Acer and Ance-distinct enzymic characteristics and alternative expression during pupal development. Eur J Biochem 257:599-606

6. Riviere G, Michaud A, Corradi HR, Sturrock ED, Ravi Acharya K, Cogez V, Bohin JP, Vieau D, Corvol P (2007) Characterization of the first angiotensin-converting like enzyme in bacteria: ancestor ACE is already active. Gene 399:81-90

7. Crackower MA, Sarao R, Oudit GY, Yagil C, Kozieradzki I, Scanga SE, Oliveira-dos-Santos AJ, da Costa J, Zhang L, Pei Y et al (2002) Angiotensin-converting enzyme 2 is an essential regulator of heart function. Nature 417:822-828

8. Gurley SB, Allred A, Le TH, Griffiths R, Mao L, Philip N, Haystead TA, Donoghue M, Breitbart RE, Acton SL et al (2006) Altered blood pressure responses and normal cardiac phenotype in ACE2-null mice. J Clin Invest 116:2218-2225

9. Camargo SM, Singer D, Makrides V, Huggel K, Pos KM, Wagner CA, Kuba K, Danilczyk U, Skovby F, Kleta R et al (2009) Tissuespecific amino acid transporter partners ACE2 and collectrin differentially interact with hartnup mutations. Gastroenterology 136:872-882

10. Kuba K, Imai Y, Rao S, Gao H, Guo F, Guan B, Huan Y, Yang P, Zhang Y, Deng W et al (2005) A crucial role of angiotensin converting enzyme 2 (ACE2) in SARS coronavirus-induced lung injury. Nat Med 11:875-879

11. Rey-Parra GJ, Vadivel A, Coltan L, Hall A, Eaton F, Schuster M, Loibner H, Penninger JM, Kassiri Z, Oudit GY et al (2012) Angiotensin converting enzyme 2 abrogates bleomycin-induced lung injury. J Mol Med. doi:10.1007/s00109-012-0859-2

12. Imai Y, Kuba K, Rao S, Huan Y, Guo F, Guan B, Yang P, Sarao R, Wada T, Leong-Poi H et al (2005) Angiotensin-converting enzyme 2 protects from severe acute lung failure. Nature 436:112-116

13. Kuba K, Imai Y, Rao S, Jiang C, Penninger JM (2006) Lessons from SARS: control of acute lung failure by the SARS receptor ACE2. J Mol Med 84:814-820 